University of Nebraska - Lincoln

DigitalCommons@University of Nebraska - Lincoln

May 2003

\title{
Finite-temperature spin polarization in half-metallic ferromagnets
}

Peter A. Dowben

University of Nebraska-Lincoln, pdowben@unl.edu

Ralph Skomski

University of Nebraska-Lincoln, rskomski2@unl.edu

Follow this and additional works at: https://digitalcommons.unl.edu/physicsdowben

Part of the Physics Commons

Dowben, Peter A. and Skomski, Ralph, "Finite-temperature spin polarization in half-metallic ferromagnets" (2003). Peter Dowben Publications. 12.

https://digitalcommons.unl.edu/physicsdowben/12

This Article is brought to you for free and open access by the Research Papers in Physics and Astronomy at DigitalCommons@University of Nebraska - Lincoln. It has been accepted for inclusion in Peter Dowben Publications by an authorized administrator of DigitalCommons@University of Nebraska - Lincoln. 


\title{
Finite-temperature spin polarization in half-metallic ferromagnets
}

\author{
P. A. Dowben and R. Skomski ${ }^{\text {a) }}$ \\ Department of Physics and Astronomy and Center for Materials Research and Analysis, \\ University of Nebraska, Lincoln, Nebraska 68588
}

(Presented on 14 November 2002)

\begin{abstract}
The temperature dependence of the spin polarization of half-metallic ferromagnets is investigated. A unitary spinor transformation shows that the corresponding spin mixing goes beyond finite-temperature smearing of the Fermi level, leading to a nonzero density of states in the gap of the insulating spin channel. As a consequence, the resistance ratio of the two spin channels changes from infinity to some finite value and, in a strict sense, half-metallic ferromagnetism is limited to zero temperature. Bloch-type spin waves and crystal imperfections contribute to the density of states in the gap but only partly explain the pronounced changes at about $0.2 T_{C}$ observed in various half-metallic magnets. In the case of NiMnSb, the spin structure depends on a nearly dispersionless transverse optical mode that occurs at about $28 \mathrm{meV}$. In terms of $3 k_{B} T$, this corresponds to 103 $\mathrm{K}$ - very close to the temperature at which there is a dramatic loss in the Ni and Mn magnetization in NiMnSb. Similar modes exist in other potential half-metallic systems. (C) 2003 American Institute of Physics. [DOI: 10.1063/1.1556132]
\end{abstract}

\section{INTRODUCTION}

The complete zero-temperature spin polarization at the Fermi level of half-metallic ferromagnets leads to an infinite resistivity of one of the spin channels, and is of considerable interest in spin electronics. ${ }^{1}$ Thermal excitations, however, deteriorate the spin polarization, ${ }^{2}$ with clear evidence of a spin-minority population far below the Curie temperature at about $0.1-0.2 T_{C}$ (spin-minority scattering) ${ }^{2-6}$. In the semiHeusler alloy NiMnSb this occurs above $80-100 \mathrm{~K}^{3-5}$ whereas in $\mathrm{CrO}_{2}$ it occurs above $50-100 \mathrm{~K}^{6}$

Far below $T_{C}$, the densities of states (DOS) of ordinary ferromagnets are only weakly modified by thermal fluctuations, because $k_{B} T$ is much smaller than the Fermi energy and band-structure distortions are small. Essentially, lowtemperature thermal disorder leads to slight modifications of the spin-dependent exchange potential and of the spin-up and spin-down densities of state. ${ }^{7,8}$ However, as elaborated on in Refs. 9 and 10, half-metallic ferromagnets are an exception, they exhibit qualitatively new low-temperature effects.

In this article, we analyze how low-temperature spin mixing affects the spin-down density of states of halfmetallic ferromagnets, discuss the experimental situation in terms of magnon-phonon interactions, and compare the changes of the spin structure with metamagnetic phase transitions.

\section{SPIN MIXING IN HALF-METALLIC FERROMAGNETS}

As discussed in Ref. 9, a simple tight-binding description of magnets with disordered moments is provided by

$$
\mathcal{H}_{\mu v i k \sigma \sigma^{\prime}}=\mathcal{T}_{\mu v i k} \delta_{\alpha \beta}-V_{\mu} \delta_{\mu v} \delta_{i k} \delta_{\alpha \beta}-I_{0} \delta_{\mu v} \delta_{i k} \mathbf{e}_{i} \sigma_{\alpha \beta} .
$$

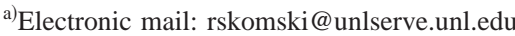

Here $\mathcal{T}_{\mu v i k}=\left\langle\phi_{\mu}\left(\mathbf{r}-\mathbf{R}_{i}\right)|\mathcal{T}| \phi_{v}\left(r-R_{k}\right)\right\rangle$ is the hopping integral between a $\mu$-type orbital at $R_{i}$ and a $\nu$-type orbital at $R_{k}, V_{\mu}$ is the crystal-field energy for the $\mu$-type orbital, $I_{0}$ is the intraatomic $d$-electron exchange, and $\mathbf{e}_{i}$ is the direction of local magnetization. In terms of Eq. (1), ferromagnetism results from the $I_{0}$ Stoner term, which reduces and enhances the energies of $\uparrow$ and $\downarrow$ electrons, respectively.

The starting point for describing finite-temperature spin disorder is the standard spin-half rotation matrix which rotates a $\uparrow$ spin by angles $\theta$ and $\phi .^{7}$ The exchange term in Eq. (1) is diagonalized by a unitary matrix $\Pi_{i} \hat{U}^{+}\left(\phi_{i}, \theta_{i}\right) \cdot{ }^{9}$ In the expression that results,

$$
\begin{aligned}
\mathcal{H}_{\mu v i k \alpha \beta}^{t}= & \mathcal{T}_{\mu v i k} \sum_{\gamma} U_{\alpha \gamma}\left(\phi_{i}, \theta_{i}\right) U_{\gamma \beta}^{+}\left(\phi_{k}, \theta_{k}\right) \\
& -\left(V_{\mu} \delta_{\alpha \beta}+I_{0} \sigma_{z \alpha \beta}\right) \delta_{\mu v} \delta_{i k},
\end{aligned}
$$

the thermal spin disorder has been mapped onto random hopping integrals. Figure 1 shows typical finite-temperature $\uparrow$ and $\downarrow$ densities of state. The effect of the spin mixing on the two subbands is indicated by the dark areas. This distortion of the $\uparrow$ DOS has the character of a quantitative correction (region I), but in the $\downarrow$ gap the DOS changes qualitatively, from zero to nonzero (region II). In other words, finitetemperature effects do not reduce to smearing of the Fermi level by a relative amount of the order of $k_{B} T / E_{F}$. As indicated in Fig. 1, the spin mixing yields small $D_{\downarrow}(E)$ values in the middle of the spin-down gap, well above the highest energy level occupied at zero temperature.

As a consequence, the resistance of the $\downarrow$ channel changes from infinity to a finite value. A crude estimate for the magnitude of this spin-mixing contribution to the DOS is

$$
D \downarrow(E) \approx \frac{M_{0}-M_{s}(T)}{M_{0}+M_{s}(T)} D_{\uparrow}(E),
$$




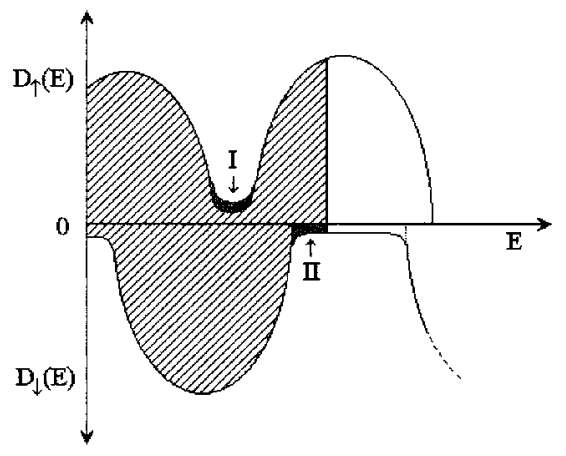

FIG. 1. Schematic $\uparrow$ and $\downarrow$ densities of states (dark shadowing denotes regions occupied at finite temperature only).

where $M_{s}(T)$ is the spontaneous magnetization and $M_{0}$ $=M_{s}(0)$. Here $M_{s}(T)$ reflects both Bloch-type spin-wave excitations and specific features such as many-sublattice effects.

\section{EXPERIMENTAL SITUATION}

Equation (1) applies not only to thermal disorder but also to structural disorder. ${ }^{711-14}$ In the case of $\mathrm{La}_{2 / 3} \mathrm{Sr}_{1 / 3} \mathrm{MnO}_{3}$, the appreciable spin minority population at $1.5 \mathrm{~K}$ is probably a band-structure effect, ${ }^{15}$ but structural features such as surfaces,${ }^{16,17}$ interfaces, ${ }^{17,18}$ defects, ${ }^{13,14}$ and substitutional disorder ${ }^{13}$ contribute to the spin-minority ( $\downarrow$ ) DOS. Note that structural disorder yields, via spin-orbit coupling, noncollinar spin structures, ${ }^{19}$ but an analog mechanism is operative in all imperfect and low-symmetry magnetic solids. ${ }^{7,20}$ However, structural disorder is essentially temperature independent, and the observed strong temperature dependence of the magnetization of half-metallic ferromagnets must be associated with structural features.

Sublattice effects associated with the compound structure of the half metallics yield low-energy transverse optical modes which couple to spin waves and reduce the net magnetization. In real space, these spin-wave modes correspond to tilting between neighboring atomic spins, with the tilt angle depending on the $k$ vector where the phonon and magnon bands cross. Generally, the closer to the Brillouin zone edge the magnons and transverse optical modes couple, the greater the tilt angle (see Fig. 2).

In the case of $\mathrm{NiMnSb}$ it is known ${ }^{21}$ that a nearly dispersionless transverse optical mode occurs at about $28 \mathrm{meV}$. In terms of $3 k_{B} T$, this corresponds to $103 \mathrm{~K}$-very close to the temperature at which there is a dramatic loss in $\mathrm{Ni}$ and Mn magnetizations in NiMnSb. ${ }^{3}$ In semi-Heusler alloys, this corresponds to tilting between neighboring $\mathrm{Ni}$ and $\mathrm{Mn}$ atoms. In real space, the mode means that the spins of Mn next neighbors form an angle of the order of $60^{\circ}$, while all the $\mathrm{Mn}$ moments are misaligned with respect to the nickel moments. ${ }^{3}$ The increase in spin-wave population in NiMnSb may also be linked to a metamagnetic phase transition between two types of ferromagnetism at about $80-100 \mathrm{~K}^{3}$

Manganese perovskites exhibit low-temperature spinwave softening and broadening of magnetoelastic origin, which is closely related to proximity of charge-ordered insulating state. ${ }^{22}$ The effect occurs where the magnon dispersion

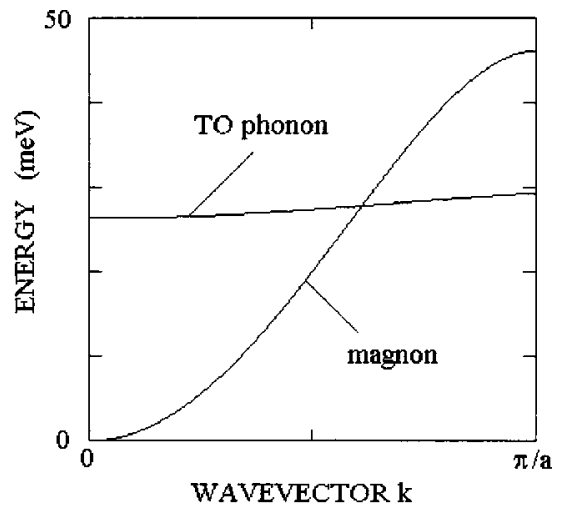

FIG. 2. Schematic of transverse optical phonon and magnon dispersions of typical half-metallic ferromagnets.

crosses the longitudinal optical branch of the phonons, at $k a / 2 \pi \approx 0.3{ }^{22,23}$ The lowest transverse optical modes in these systems, about $23 \mathrm{meV}$ for $\mathrm{La}_{0.7} \mathrm{~A}_{0.3} \mathrm{MnO}_{3}$ ( $\mathrm{A}$ $=\mathrm{Ca}, \mathrm{Sr}),{ }^{22}$ suggest metamagnetic transitions, similar to those observed in NiMnSb, ${ }^{3}$ at about $84 \mathrm{~K}$. The tunnel magnetoresistance of $\mathrm{La}_{1-x} \mathrm{Sr}_{x} \mathrm{MnO}_{3} / \mathrm{SrTiO}_{3} / \mathrm{La}_{1-x} \mathrm{Sr}_{x} \mathrm{MnO}_{3}$ where $x=0.2$ and 0.3 dramatically drops at about 27 (Ref. $24)$ and $130 \mathrm{~K},{ }^{25}$ respectively. This suggests a loss of net spin polarization, ${ }^{10}$ although Coulomb-blockade contributions ${ }^{26}$ have not been eliminated from these experiments.

\section{DISCUSSION AND CONCLUSIONS}

The appearance of the spin-down density (region II) in Fig. 1 has a simple physical interpretation: a $\uparrow$ electron hops onto a neighboring atomic site characterized a different axis of quantization. In the frame of the neighboring atom, the spin wave function has nonzero projections onto both $\uparrow$ and $\downarrow$ states. For random disorder, the spin mixing can be interpreted as Anderson localization of spin-polarized $3 d$ electrons in a thermally randomized atomic potential. The specific situation encountered in half-metallic ferromagnets reflects the many-sublattice character of the compounds. This leads to the above-mentioned magnon-phonon coupling, also observed in Ref. 22. Note that similar phonon modes exist in a variety of compounds, including materials such as $\mathrm{CrO}_{2}$ and $\mathrm{Fe}_{3} \mathrm{O}_{4}$. The finite- $k$ spin disorder is reminiscent of a metamagnetic phase transition into a noncollinear state, and the states in the spin-down gap (region II in Fig. 1) reflect the presence of partly or completely reversed spins in the thermally excited state.

The present findings are relevant to the question of whether and under what circumstances there exists the "ideal," that is, fully spin-polarized half-metallic, system. There have been various arguments in favor of ${ }^{27-33}$ and against ${ }^{3,9,10,15-17}$ true half-metallic ferromagnetism. In our opinion, half-metallic ferromagnetism is an idealized limit, realistic only in perfect crystals at zero temperature. For example, even though tunnel junctions include contributions from the spin-dependent Fermi velocity, ${ }^{34}$ which could effectively enhance tunnel magnetoresistance,,${ }^{15,34}$ no tunnel magnetoresistive junctions measurement has provided a strong indication of the half-metallic character. The highest tunnel 
magnetoresistance values reached have been as high as $450 \%$ (Ref. 35) $-500 \%,{ }^{36}$ and this only at very low temperatures and still well below the values expected for a half metal. Transport spin-polarization measurements using pointcontact Andreev reflection have provided the highest measured polarizations to date. ${ }^{15,30,37,38}$ In spite of one claim of proof of half-metallic character, ${ }^{30}$ these measurements also miss the mark of $100 \%$ polarization for the postulated halfmetallic systems, ${ }^{15,30,37,38}$ even at temperatures of $1.6 \mathrm{~K}$.

In conclusion, we have shown that finite-temperature spin disorder destroys the complete spin-polarization characteristic of half-metallic ferromagnets. The many-sublattice structure of the half-metallic ferromagnets yields transverse optical phonon modes which couple to the magnons and tend to reduce the net magnetization and the Fermi-level spin polarization at comparatively low temperatures. In a strict sense, this thermally activated spin mixing means that halfmetallic ferromagnetism is limited to perfect crystals at zero temperature. This mechanism is modified and, in general, enhanced by crystal imperfections, and by surface and interface effects.

Note added in proof: After the submission of this paper, the authors became aware that a previous publication on magnetic nanojunctions ${ }^{39}$ anticipates some features of the present approach.

\section{ACKNOWLEDGMENTS}

This work was supported by NSF (Grant No. DMR-9802126), CMRA, and Office of Naval Research.

${ }^{1}$ W. E. Pickett and J. Moodera, Phys. Today 54, 39 (2001).

${ }^{2}$ J. M. D. Coey and M. Venkatesan, J. Appl. Phys. 91, 8345 (2002).

${ }^{3}$ C. N. Borca et al., Phys. Rev. B 64, 052409 (2001).

${ }^{4}$ Ch. Hordequin, E. Lelievre-Berna, and J. Pierre, Physica B 234-236, 602 (1997).

${ }^{5}$ Ch. Hordequin, D. Ristoiu, L. Ranno, and J. Pierre, Eur. Phys. J. B 16, 287 (2000).

${ }^{6}$ S. M. Watts, S. Wirth, S. von Molnár, A. Barry, and J. M. D. Coey, Phys. Rev. B 61, 9621 (2000).

${ }^{7}$ L. M. Sandratskii, Adv. Phys. 47, 91 (1998).

${ }^{8}$ L. M. Sandratskii and J. Kübler, in Magnetism and Electronic Correlations, in Local Moment Systems, edited by M. Donath, P. A. Dowben, and W. Nolting (World Scientific, Singapore 1998), Fig. 6, p. 271.
${ }^{9}$ R. Skomski and P. A. Dowben, Europhys. Lett. 58, 544 (2002).

${ }^{10}$ H. Itoh, T. Ohsawa, and J. Inoue, Phys. Rev. Lett. 84, 2501 (2000).

${ }^{11}$ R. Lorenz, J. Hafner, S. S. Jaswal, and D. J. Sellmyer, Phys. Rev. Lett. 74, 3688 (1995).

${ }^{12}$ R. Skomski and J. M. D. Coey, Permanent Magnetism (Institute of Physics, Bristol, 1999).

${ }^{13}$ D. Orgassa, H. Fujiwara, T. C. Schulthess, and W. H. Butler, Phys. Rev. B 60, 13237 (1999).

${ }^{14}$ D. Orgassa, H. Fujiwara, T. C. Schulthess, and W. H. Butler, J. Appl. Phys. 87, 5870 (2000)

${ }^{15}$ B. Nadgorny et al., Phys. Rev. B 63, 184433 (2001).

${ }^{16}$ S. J. Jenkins and D. A. King, Surf. Sci. Lett. 494, L793 (2001).

${ }^{17}$ G. A. de Wijs and R. A. de Groot, Phys. Rev. B 64, 020402 (2001)

${ }^{18}$ S. J. Jenkins and D. A. King, Surf. Sci. Lett. 501, L185 (2002).

${ }^{19}$ K.-H. Fischer and A. J. Hertz, Spin Glasses (Cambridge University Press, Cambridge, UK, 1991).

${ }^{20}$ L. M. Sandratskii, Phys. Rev. B 64, 134402 (2001).

${ }^{21}$ C. N. Borca, D. Ristoiu, T. Komesu, H. Jeong, Ch. Hordequin, J. Pierre, J. P. Nozières, and P. A. Dowben, Appl. Phys. Lett. 77, 88 (2000).

${ }^{22}$ P. Dai, H. Y. Hwang, J. Zhang, A. Fernandez-Baca, S.-W. Cheong, C. Kloc, Y. Tomioka, and Y. Tokura, Phys. Rev. B 61, 9553 (2000).

${ }^{23}$ L. M. Woods, Phys. Rev. B 65, 014409 (2000).

${ }^{24}$ T. Obata, T. Manako, Y. Shimakawa, and Y. Kubo, Appl. Phys. Lett. 74, 290 (1999).

${ }^{25}$ J. Z. Sun, Philos. Trans. R. Soc. London, Ser. A 356, 1693 (1998).

${ }^{26}$ A. Sokolov et al., Europhys. Lett. 58, 448 (2002).

${ }^{27}$ J.-H. Park, E. Vescovo, H.-J. Kim, C. Kwon, R. Ramesh, and T. Venkatesan, Nature (London) 392, 794 (1998).

${ }^{28}$ K. P. Kämper, W. Schmitt, G. Güntherodt, R. J. Gambino, and R. Ruf, Phys. Rev. Lett. 59, 2788 (1987)

${ }^{29}$ Y. S. Dedkov, M. Fonine, C. Konig, U. Rüdiger, G. Güntherodt, S. Senz, and D. Hesse, Appl. Phys. Lett. 80, 4181 (2002).

${ }^{30}$ Y. Ji, G. J. Strijkers, F. Y. Yang, C. L. Chien, J. M. Byers, A. Anguelouch, G. Xiao, and A. Gupta, Phys. Rev. Lett. 86, 5585 (2001).

${ }^{31}$ R. Wiesendanger, H.-J. Güntherodt, G. Güntherodt, R. J. Gambino, and R. Ruf, Phys. Rev. Lett. 65, 247 (1990).

${ }^{32}$ Y. S. Dedkov, U. Rüdiger, and G. Güntherodt, Phys. Rev. B 65, 064417 (2002).

${ }^{33}$ J. Y. T. Wei, N. C. Yeh, and R. P. Vasquez, Phys. Rev. Lett. 79, 5150 (1997).

${ }^{34}$ I. I. Mazin, Phys. Rev. Lett. 83, 1427 (1999).

${ }^{35}$ M. Viret, J. Nassar, M. Drouet, J. P. Contour, C. Fermon, and A. Fert, J. Magn. Magn. Mater. 198-199, 1 (1999).

${ }^{36}$ J. J. Versluijs, M. A. Bari, and J. M. D. Coey, Phys. Rev. Lett. 87, 6601 (2001).

${ }^{37}$ R. J. Soulen et al., Science 282, 85 (1998).

${ }^{38}$ W. J. DeSisto, P. R. Broussard, T. F. Ambrose, B. E. Nadgorny, and M. S. Osofsky, Appl. Phys. Lett. 76, 3789 (2000).

${ }^{39}$ A. H. MacDonald, T. Jungwirth, and M. Kasner, Phys. Rev. Lett. 81, 705 (1998) 